S93 PULMONARY MATRIX METALLOPROTEINASES AND SMALL AIRWAYS DISEASE IN COPD - THE ORIGINS OF AIRFLOW OBSTRUCTION?

${ }^{1} \mathrm{~K}$ Ostridge, ${ }^{1} \mathrm{~N}$ Williams, ${ }^{1} \mathrm{~V}$ Kim, ${ }^{1} \mathrm{M}$ Bennett, ${ }^{2} \mathrm{~S}$ Harden, ${ }^{1} \mathrm{~L}$ Welch, ${ }^{3} \mathrm{~S}$ Bourne, ${ }^{4} \mathrm{~N}$ Coombs, ${ }^{3} \mathrm{P}$ Elkington, ${ }^{3} \mathrm{~K}$ Staples, ${ }^{3} \mathrm{~T}$ Wilkinson. ${ }^{1}$ Southampton NIHR Respiratory Biomedical Research Unit, Southampton General Hospital, Southampton, UK; ${ }^{2}$ Southampton General Hospital, Southampton, UK; ${ }^{3}$ Clinical and Experimental Sciences, University of Southampton Faculty of Medicine, Southampton, UK; ${ }^{4}$ Primary Care and Population Sciences, University of Southampton Faculty of Medicine, Southampton, UK

\subsection{6/thoraxinl-2015-207770.99}

Introduction and objectives Matrix-metalloproteinases (MMPs) are proteolytic enzymes that can degrade the extra-cellular matrix (ECM) and drive tissue remodelling, key processes in the pathogenesis of COPD. The development of small airway disease and emphysema have been identified as critical mechanisms in the development of airflow obstruction but the contribution of MMPs in human disease is poorly characterised.

We investigated the role of MMPs in the lung by quantifying levels and determining relationships with the key pathological components of COPD, measured by CT, in patients and healthy controls.

Methods 24 mild and moderate COPD and 8 control subjects were enrolled onto the study and underwent bronchoalveolar lavage (BAL) and high resolution CT. We analysed levels of MMPs in BAL using a Luminex immunoassay. Image analysis, performed using VIDA Apollo software, quantitatively assessed emphysema, bronchial wall thickening and small airways disease. Results Multiple MMPs (MMP-1, -2, -3, -8, -9 and -10) were significantly elevated in the lungs of COPD subjects. MMP -3, $7,-8,-9,-10$, and -12 concentrations were closely associated with CT markers of small airways disease (Table 1). Emphysema severity was also associated with MMP-3, $-7,-8$ and -10 . However there were no strong relationships between MMPs and bronchial wall thickness of the larger airways.

Stepwise linear regression analysis identified MMP-10 to be the only significant predictor of emphysema $\left(R^{2} 0.34, p 0.001\right)$ that was independent of each of the other MMPs while MMP-8 was the only significant predictor of small airways disease $\left(\mathrm{R}^{2}\right.$ $0.56, \mathrm{p}<0.001)$ independent of each of the other MMPs.

Conclusion Pulmonary MMP concentrations are directly associated with the extent of gas trapping and small airways disease identified on CT scan. This suggests that MMPs may play a significant role in the pathogenesis of COPD by causing breakdown of the pulmonary ECM leading to abnormal remodelling in both the small airways and lung parenchyma. Whilst most previous work has focused on MMPs and emphysema, this study shows the strongest associations were with small airways disease.
594 THE ROLE OF OXIDATIVE STRESS AND ANTIOXIDANTS IN SEVERE ASTHMA; A CASE CONTROL STUDY

A Bishopp, R Sathyamurthy, AH Mansur. Birmingham Regional Severe Asthma Service, Heartlands Hospital, Birmingham, UK

\subsection{6/thoraxjnl-2015-207770.100}

Background A number of antioxidant defences counteract potentially damaging oxidising species within the respiratory tract. Many enzymes, such as the superoxide dismutases and glutathione peroxidases, require essential elements to maintain function. Vitamins $\mathrm{A}, \mathrm{C}$ and $\mathrm{E}$ also have antioxidant properties. It has been suggested that asthmatic patients are deficient in antioxidants. This study assessed levels of serum antioxidants, markers of airways inflammation and resultant oxidative stress burden with increasing severity of asthma.

Method This is a case control study comparing patients with severe asthma (BTS steps 4/5) to mild corticosteroids-naïve asthmatics and normal healthy controls. Participants were assessed in terms of asthma diagnosis, severity, exhaled breath condensate (EBC) nitrite, fractional exhaled nitric oxide (feNO), spirometry, and serum copper, zinc, selenium, vitamin $\mathrm{A}$ and vitamin $\mathrm{E}$ measurements. Analyses were conducted using Kruskal-Wallis, one way analysis of variance.

Results The severe asthma group was comprised of 30 patients (23 females, mean age 41.4 years [range 19 to 56], mean FEV1 2.2 litres [\% predicted $72.2 \%$ ], mean $\mathrm{FEV} 1 / \mathrm{FVC}$ ratio $72.7 \%$, mean daily inhaled corticosteroid equivalent to 2,390 $\mu \mathrm{g} \mathrm{BDP}$, with $17 / 30$ [57\%] on maintenance oral corticosteroids). Fifteen mild and corticosteroid naïve patients were recruited ( 9 females, mean age 34.6 years [range 19-57], mean FEV1 3.481 [\% predicted 100.5\%], FEV1/FVC ratio 82.2\%). All 15 were treated with salbutamol only. The control group was comprised of 15 patients (12 females, mean age 37.6 years [range 25-52], mean FEV1 3.53 [\%predicted 111.7\%], and FEV1/FVC ratio 81.9\%). FeNO increased significantly with increasing severity $(\mathrm{p}=$ $0.001)$, but EBC nitrite did not $(\mathrm{p}=0.9)$. Vitamin $\mathrm{A}$ and $\mathrm{E}$ levels increased significantly with increased disease severity $(\mathrm{p}=$ 0.04 and 0.0008 respectively). There was no significant difference between groups with copper $(\mathrm{p}=0.98)$, zinc $(\mathrm{p}=0.8)$ or selenium $(\mathrm{p}=0.9)$.

Conclusion We observed no evidence of deficiency in the serum minerals or vitamins in the severe asthmatics with increased FeNO but not EBC nitrite. We conclude that impaired oxidative stress defences in severe asthma may be driven by factors other than vitamin or mineral deficiency.

\begin{tabular}{|c|c|c|c|c|c|c|c|c|c|}
\hline & MMP-1" & MMP-2 ${ }^{\#}$ & MMP-3\# & MMP-7\# & MMP-8 ${ }^{\#}$ & MMP-9\# & MMP-10 & MMP-12 & MMP-13 \\
\hline Emphysema\% (LAA\%) & 0.01 & 0.02 & $0.25^{* *}$ & $0.23^{* *}$ & $0.15^{*}$ & 0.11 & $0.34^{* *}$ & 0.01 & 0.01 \\
\hline Small airways Disease (E/l MLD) & 0.14 & 0.14 & $0.53^{* * *}$ & $0.29^{*}$ & $0.56^{* * *}$ & $0.36^{* *}$ & $0.50 * * *$ & $0.24^{*}$ & 0.00 \\
\hline Bronchial wall area (Pi10) & 0.03 & 0.00 & 0.00 & 0.01 & 0.04 & 0.01 & 0.00 & $0.17^{*}$ & 0.00 \\
\hline
\end{tabular}

$\mathrm{R}^{2}$ values given. "These values were logged to improve normality of residuals. LAA\% $(n=31)$, Pi10 $(n=31)$ and E/l MLD $(n=22) .{ }^{*} p<0.05{ }^{* *} p<0.01{ }^{* * *} p<0.001$. 

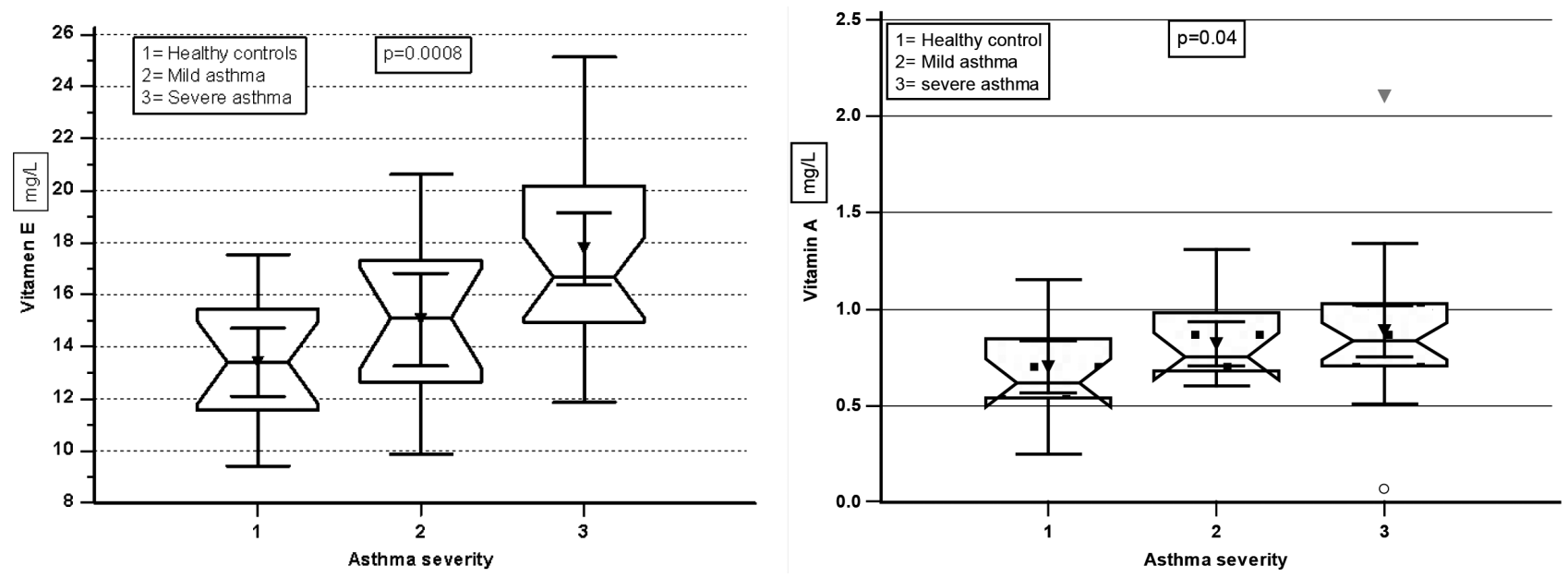

Abstract S94 Figure 1 Vitamin levels across severity groups

\section{S95 PERIPHERAL BLOOD CRTH2 POSITIVE CELL COUNT IN PATIENTS WITH SEVERE EOSINOPHILIC ASTHMA}

${ }^{1} \mathrm{~B}$ Hilvering, ${ }^{2} \mathrm{~L}$ Stoeger, ${ }^{2} \mathrm{~S}$ Go, ${ }^{2} \mathrm{C}$ Connelly, ${ }^{2} \mathrm{~K}$ Borg, ${ }^{2} \mathrm{~S}$ Thulborn, ${ }^{2} \mathrm{~S}$ Pahlke, ${ }^{2} \mathrm{ID}$ Pavord, ${ }^{2} \mathrm{~L}$ Xue. ${ }^{1}$ University of Utrecht, Utrecht, Netherlands; ${ }^{2}$ Univesity of Oxford, Oxford, UK

\subsection{6/thoraxjnl-2015-207770.101}

Background CRTH2 antagonism has been shown to reduce eosinophilic airway inflammation and improve lung function in patients with severe eosinophilic asthma. To better understand the role of CRTH2 in the pathogenesis of this asthma phenotype, we have carried out a cross-sectional study to investigate the CRTH2 positive cell counts in peripheral blood of patients with severe eosinophilic asthma.

Methods Blood was taken from 12 controls and 33 patients with asthma, 21 of whom met the 2014 ERS/ATS guideline criteria for severe asthma and had historical evidence of eosinophilic airway inflammation as defined before (Pavord et al. Lancet 2012;380:651-9). Th2 were detected as $\mathrm{CD}^{+}{ }^{+} \mathrm{CD} 4^{+} \mathrm{CRTH} 2+$, $\mathrm{Tc} 2$ as $\mathrm{CD}^{+}{ }^{+} \mathrm{CD} 8{ }^{+} \mathrm{CRTH} 2+$, eosinophils as $\mathrm{SSC}^{\text {high }} \mathrm{CRTH} 2+$, and basophils as $\mathrm{CD} 123^{+} \mathrm{CRTH} 2+$ by flow cytometry, and numbers presented as total cell counts in peripheral blood. Data were analysed using one-way ANOVA followed by the NewmanKeuls test.
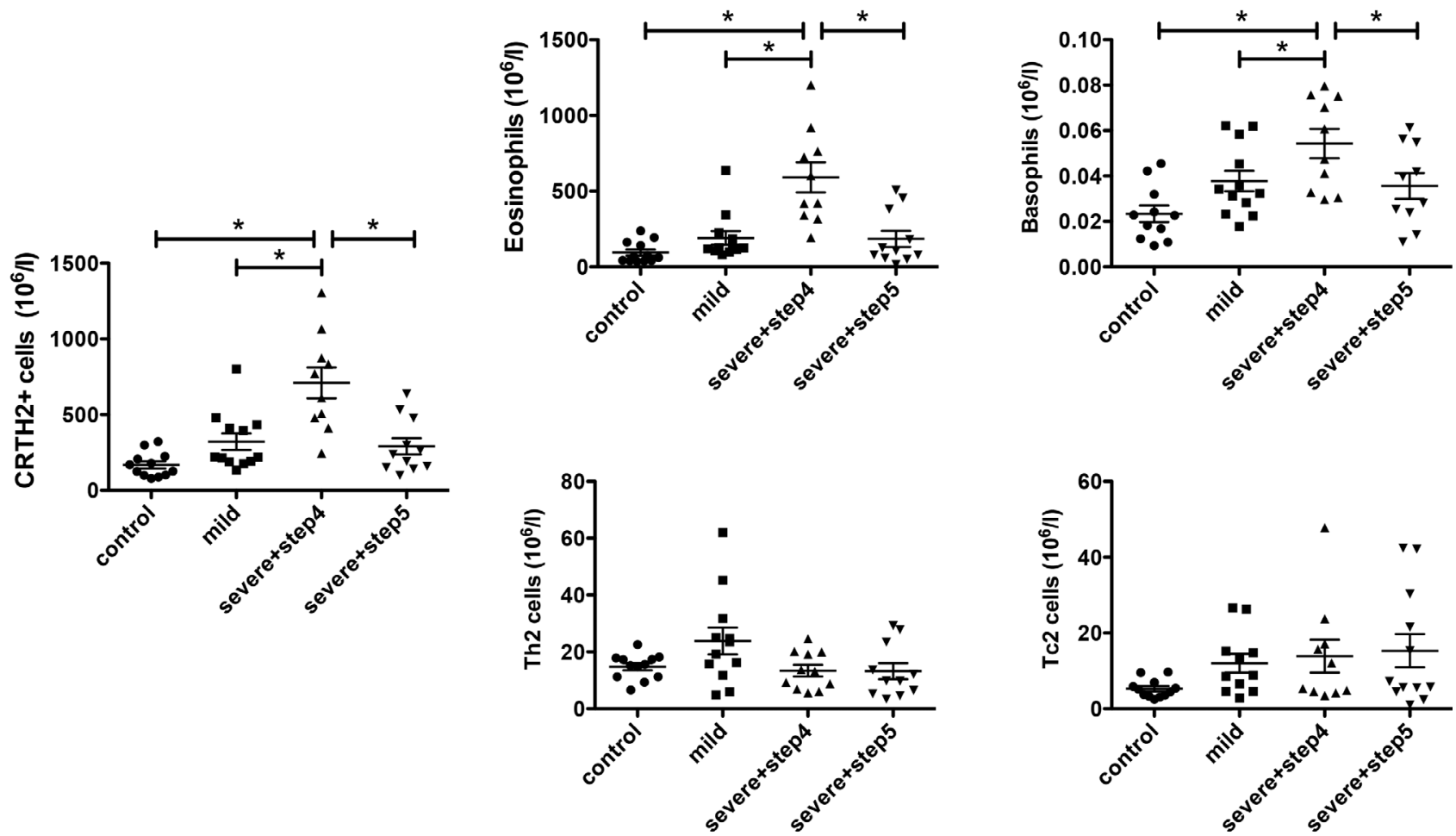

Abstract S95 Figure 1 Comparison of eosinophil, basophil, Th2, Tc2 and total CRTH2+ cell counts in the blood from healthy control and different asthma patients. ( $p<0.0001$ for CRTH2+ and eosinophils; $p<0.001$ for basophils; $p<0.05$ for Th2; and $p<0.1$ for Tc2. * $p<0.05$ between the indicated groups) 